\title{
Correlation between clinical and pathologic diagnosis in oral leukoplakia in 54 patients
}

\author{
Rocío Vázquez-Álvarez ${ }^{1}$, Francisca Fernández-González ${ }^{1}$, Pilar Gándara-Vila ${ }^{2}$, Dolores Reboiras-López ${ }^{2}$, \\ Abel García-García ${ }^{3}$, José-Manuel Gándara-Rey ${ }^{4}$
}

\begin{abstract}
${ }^{1}$ Dental surgeon. Student at the Master Degree Course in Oral Medicine, Oral Surgery, and Implantology. Faculty of Medicine and Odontology at the Universidad de Santiago de Compostela

${ }^{2}$ Dental surgeon. Lecturer at the Master Degree Course in Oral Medicine, Oral Surgery, and Implantology. Faculty of Medicine and Odontology at the Universidad de Santiago de Compostela

${ }^{3}$ Maxillofacial surgeon. Tenured lecturer in oral surgery. Director of the Master Degree Course in Oral Medicine, Oral Surgery, and Implantology. Faculty of Medicine and Odontology at the Universidad de Santiago de Compostela. Head of Maxillofacial Surgery Department at Hospital Provincial de Santiago de Compostela

${ }^{4}$ Stomatologist. Professor in oral medicine. Director of the Master Degree Course in Oral Medicine, Oral Surgery, and Implantology. Faculty of Medicine and Odontology at the Universidad de Santiago de Compostela
\end{abstract}

Correspondence:

Facultad de Odontología

c/ Entrerrios $s / n$

15705 Santiago de Compostela,

A Coruña, Spain

josemanuel.gandara@usc.es

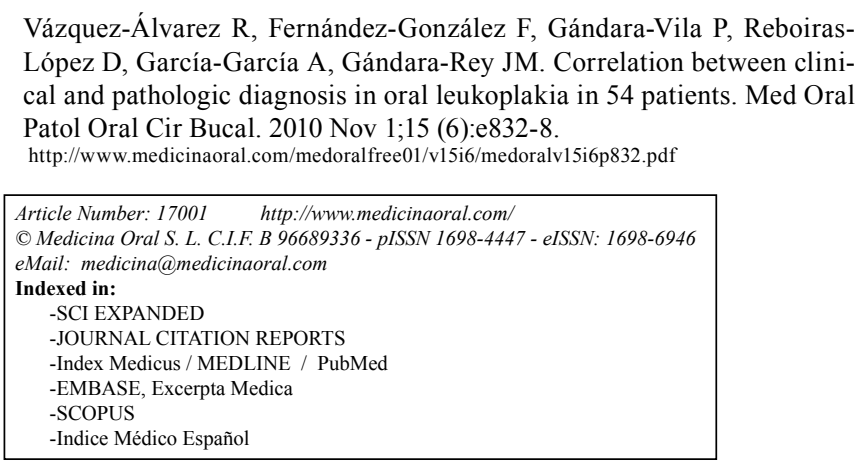

\begin{abstract}
The main aim of this study was to establish a correlation between the clinical and pathologic diagnosis of oral leukoplakia with a particular focus on epithelial dysplasia. We reviewed the medical records of 54 patients with a clinical and histologic diagnosis of oral leukoplakia who were seen at our center between 2002 and 2008. We found that the disease was more common in men (59.3\%) than in women and we also detected a significantly greater prevalence of alcohol and tobacco consumption in men. The mean age of the patients was 62.57 years. Three patients had been histologically diagnosed with invasive cancer and 4 with carcinoma in situ. The most common lesion site for leukoplakias with severe dysplasia and invasive carcinoma was the lateral aspect of the tongue, the floor of the mouth, and the gums. It is therefore essential to include these sites in the clinical examination to aid early diagnosis. A higher degree of dysplasia should be suspected in non-homogeneous leukoplakias. While dysplasia is associated with a greater risk of malignant transformation, it is also important to monitor leukoplakias without dysplastic features as they can occasionally be the site of carcinoma.
\end{abstract}

Key words: Oral leukoplakia, epithelial dysplasia, oral cancer. 


\section{Introduction}

Based on work by the Pindborg school, the World Health Organization (WHO) defined leukoplakia in 1978 as a white patch or plaque that cannot be rubbed off or characterized as any other disease. The WHO also stated that it was not a histopathologic entity as it was characterized by a wide variety of microscopic alterations. This essentially clinical definition of leukoplakia still holds today. At the first international conference on leukoplakia held in Malmö, Sweden in June 1983, it was decided to refine the definition by adding that the condition was not associated with any chemical or physical causative agents except tobacco.

In 1994, the WHO defined oral leukoplakia as a predominantly white lesion of the oral mucosa that cannot be characterized as any other definable lesion. Two years later, Axell et al (1) extended this definition by adding that some oral leukoplakias can transform into cancer. In a more recent publication, leukoplakia was defined as "white plaques of questionable risk having excluded (other) known diseases or disorders that carry no increased risk for cancer" (2).

Nowadays, certain authors recommend that an initial clinical diagnosis of leukoplakia should be considered provisional and confirmed histologically if the lesion persists after 2-4 weeks and when all other possible etiologic risk factors have been ruled out (3).

Leukoplakia shows characteristic histologic findings such as epithelial hyperplasia, hyperkeratosis, and/or hyperparakeratosis, with or without epithelial dysplasia or carcinoma. It is the most common precancerous lesion of the oral cavity. Leukoplakia and erythroplasia are the 2 precancerous lesions par excellence of the oral mucosa. The risk of malignant transformation of leukoplakia with dysplasia has been reported to be high as $43 \%$ (4), hence the need for regular monitoring.

Increased malignant potential may be associated with certain clinical characteristics such as lesion type, size, and site, dysplasia, and tobacco use. Nevertheless, no clear correlation has been found between clinical and pathologic diagnosis because seemingly similar lesions may be histologically different. This highlights the need for epidemiological studies to determine clinical and histopathologic characteristics of leukoplakia that shed light on the behavior of these lesions and contribute to the early detection of oral cancer (3).

\section{Objectives}

1. To determine the most common lesion sites and clinical oral forms in a series of 54 patients and determine possible etiologic factors.

2. To establish a correlation between the clinical and pathologic diagnosis of oral leukoplakia, with a focus on epithelial dysplasia.

\section{Materials and Methods}

We reviewed the clinical records of 54 patients who were diagnosed with oral leukoplakia both clinically and histologically at the unit attached to the Master Degree Course in Oral Medicine, Oral Surgery, and Implantology at Universidad de Santiago de Compostela, Spain, between 2002 and 2008. All the biopsy samples had been preserved in $10 \%$ formaldehyde and sent to the pathology department at Hospital Clínico Universitario de Santiago de Compostela, where they were analyzed by the same pathologist.

We analyzed the following variables: sex, age, place of residence (urban vs rural environment, defined as having fewer than 2000 inhabitants), personal and family history of cancer, tobacco and alcohol use, mouthwash use, concomitant systemic diseases, response to previous treatment, lesion site, presence of single or multiple lesions, lesion surface, symptoms, time since onset, treatment, type of leukoplakia, lesion color and size (largest diameter), keratosis, and dysplasia.

All the data were analyzed using version 15.0 of SPSS for Windows. Statistical significance was set at a $p$ value of $<.05$.

\section{Results}

Oral leukoplakia lesions were more common in men than in women (59.3\% vs $40.7 \%)$, which corresponds to a male to female ratio of 1.45:1.

The mean (SD) age of the patients was 62.57 (12.75) years, with a range of 33 to 92 years.

The majority of patients (79.6\%) were aged between 50 and 79 years (Fig. 1).

As can be seen in figure 2, there was a tendency for oral leukoplakia to present earlier in men (mean age, 60 years) than in women (mean age, 66.27 years).

Almost two-thirds of the patients (63\%) lived in an urban environment, contrasting with $37 \%$ who lived in a rural environment.

A personal and family history of cancer was detected in $7.4 \%$ and $41.7 \%$ of the patients, respectively.

On analyzing smoking, we found that $48.1 \%$ of the patients were non-smokers, $33.3 \%$ were smokers, and $18.5 \%$ were ex-smokers. Within the group of smokers, $50 \%$ smoked black tobacco and a slightly lower percentage (44.4\%) smoked blond tobacco. A single patient smoked a pipe.

Most smoked more than 20 cigarettes a day. The second largest group smoked 10-20 cigarettes a day, and the smallest group smoked fewer than 10 cigarettes a day. None of the ex-smokers had smoked fewer than 10 cigarettes a day and the majority had smoked 10- 20 cigarettes a day.

All the smokers had been smoking for more than 10 years, with a majority $(29.6 \%)$ reporting a smoking history of more than 20 years. Most of the ex-smokers had 


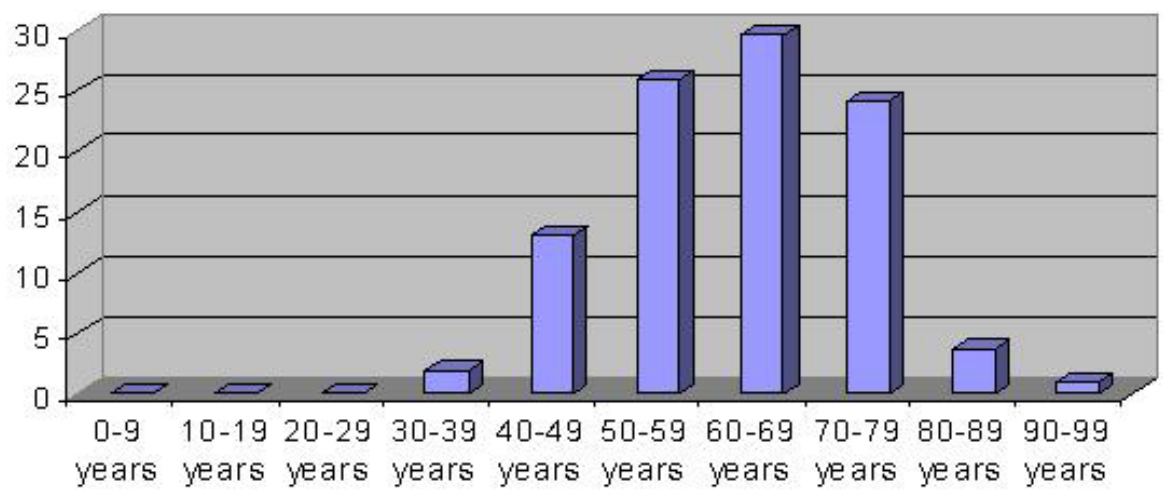

Fig. 1. Percentage distribution of leukoplakia by age group.

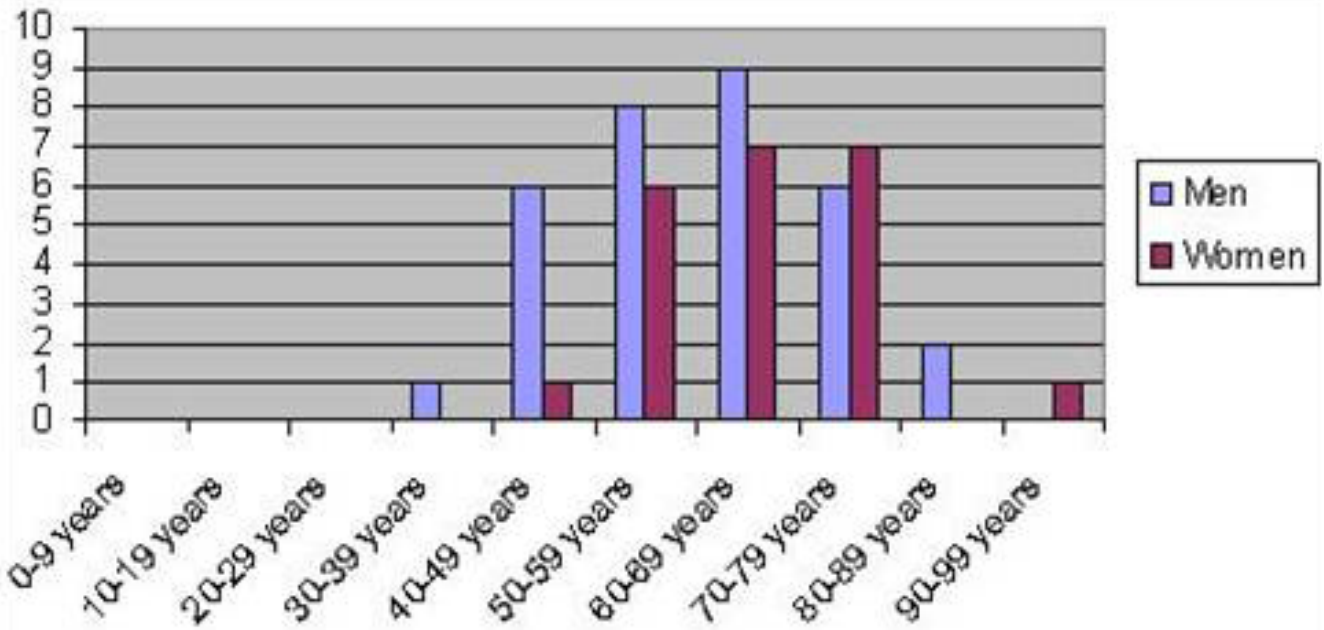

Fig. 2. Association between age and sex.

quit over 10 years previously. The next largest group had quit between 1 and 4 years earlier, followed by those who had quit between 5 and 9 years earlier. Only 1 patient had quit the habit in the preceding year.

We found that patients who smoked black tobacco smoked more than those who smoked blond tobacco. In the black tobacco group, for example, $77.8 \%$ smoked more than 20 cigarettes and the remaining $22.2 \%$ smoked 10-20 cigarettes a day. In the blond tobacco group, 62.5\% smoked between 10 and 20 cigarettes a day, 25\% smoked fewer than 10 cigarettes a day, and just $12.5 \%$ smoked more than 20 cigarettes a day. All of the patients who smoked black tobacco $(n=9)$ had been smoking for more than 20 years. Of those who smoked blond tobacco, $75 \%$ had been smoking for more than 20 years and $25 \%$ had started between 10 and 20 years previously.
Over half of the group (55.6\%) did not drink alcohol; $1.9 \%$ drank occasionally, 33.3\% drank less than $500 \mathrm{cc}$ a day, 5.6\% drank more than $500 \mathrm{cc}$ day, and the remaining $3.7 \%$ were ex-alcoholics. Almost all of those who drank $(90.9 \%)$ drank wine and/or beer only. The remaining 9.1\% drank wine, beer, and spirits. Patients who smoked the most were also regular drinkers (significant association, $\mathrm{p}=.01$ ).

The most common systemic diseases were hypercholesterolemia and rheumatic diseases (both detected in $16.7 \%$ of the sample).

The lateral aspect of the tongue was the most common lesion site $(25.9 \%$ of patients), followed by the gums $(18.5 \%)$, the buccal mucosa $(16.7 \%)$, the floor of the mouth $(9.3 \%)$ the palate, lip, and ventral surface of the tongue (each $7.4 \%$ ), and, finally, the dorsal surface of the tongue and behind the commissures (each, 3.7\%). The 
most common sites in younger patients were the commissure areas (mean age, 44 years) and the palate (mean age, 53.5 years). In elderly patients, the most common sites were the dorsal surface of the tongue (mean age, 72 years), the lip (mean age, 66 years), and the floor of the mouth (65.2 years). The most common lesion sites in men were the buccal mucosa and the floor of the mouth; in women, they were the lateral aspect of the tongue and the gums. The lips were equally affected in men and women. The association between sex and lesion site was not statistically significant $(\mathrm{p}>.05)$. More patients had single lesions than multiple lesions (61.1\% vs 39.9\%).

Almost half (48.1\%) of the patients did not remember when the lesions had appeared; $31.5 \%$ reported a time since onset of over 12 months. The other times since onset reported were 3 to 6 months $(9.3 \%)$, under 3 months (7.4\%), and 6 to 12 months (3.7\%).

Homogeneous leukoplakia was the most common type of leukoplakia detected (present in $51.9 \%$ of patients), followed by verrucous leukoplakia (35.2\%), erythroleukoplakia (9.3\%), and nodular leukoplakia (3.7\%). One of the 2 patients with erythroleukoplakia had mottled leukoplakia. The most common sites by type of leukoplakia were the lateral aspect of the tongue for homogeneous leukoplakia, the gums for verrucous leukoplakia, and the gums and behind the commissures for nodular leukoplakia. The buccal mucosa was involved in the patient with mottled leukoplakia.

The mean (SD) lesion size was $2.2(1.54) \mathrm{cm}$. Based on the classification system proposed by Axéll et al (1), half of the lesions were L1 lesions $(<2 \mathrm{~cm}), 38.9 \%$ were L2 lesions $(2-4 \mathrm{~cm})$, and the remaining $11.1 \%$ were L3 lesions $(>4 \mathrm{~cm})$.
On reviewing the histopathologic results, we observed that $74.1 \%$ of lesions had no dysplastic features; $93 \%$ had mild dysplastic features, 3.7\% had moderate dysplastic features, $7.4 \%$ had severe dysplastic features or carcinoma in situ, and 5.6\% had invasive carcinoma. Degree of dysplasia was significantly associated with type of leukoplakia ( $p=.007)$ (Table 1) but not with lesion size.

Dysplasia was significantly more common in patients with a family history of cancer than in those without $(p=.02)$ but we found no association between degree of dysplasia and personal history of cancer. We observed a trend, without statistical significance, towards a predominance of no dysplastic features in younger patients. All of the cases of invasive carcinoma were in patients in their 60s. Although also without statistical significance, lesions with moderate or severe dysplasia or invasive carcinoma were more common in women, something which is worth considering as a possible marker for poor prognosis (Fig. 3).

The association between lesion site and degree of dysplasia was not statistically significant (Table 2). We also found no association between clinical surface and degree of dysplasia. It is noteworthy that only 1 of the 3 carcinomas diagnosed caused pain.

No significant association was found between lesion size and degree of dysplasia. According to the staging system proposed by van der Waal \& Axéll (3) van der Waal et al (5), 38.9\% of the lesions were stage I, 33.3\% were stage III, $11.1 \%$ were stage II, and $3.7 \%$ were stage IV. Of the 54 patients analyzed, 7 had carcinoma, 4 of which were staged as TisNoMo and 3 of which were staged as T1NoMo.

Table 1. Comparison of clinical type of leukoplakia and degree of dysplasia

\begin{tabular}{|c|c|c|c|c|c|c|}
\hline \multirow[t]{2}{*}{ Degree of dysplasia } & \multicolumn{5}{|c|}{ Clinical type } & \multirow[b]{2}{*}{ Total } \\
\hline & $\begin{array}{l}\text { Homogenous } \\
\text { leukoplakia }\end{array}$ & $\begin{array}{c}\text { Non- } \\
\text { homogeneous } \\
\text { leukoplakia }\end{array}$ & $\begin{array}{l}\text { Nodular } \\
\text { leukoplakia }\end{array}$ & Erythroleukoplakia & $\begin{array}{c}\text { Mottled } \\
\text { leukoplakia }\end{array}$ & \\
\hline None & $24(44.4 \%)$ & $13(24.1 \%)$ & $2(3.7 \%)$ & - & $1(1.9 \%)$ & $40(74.1 \%)$ \\
\hline Mild & $3(5.6 \%)$ & $2(3.7 \%)$ & - & - & - & $5(9.3 \%)$ \\
\hline Moderate & $1(1.9 \%)$ & $1(1.9 \%)$ & - & - & - & $2(3.7 \%)$ \\
\hline Severe dysplasia or & - & $2(3.7 \%)$ & - & $2(3.7 \%)$ & - & $4(7.4 \%)$ \\
\hline \multicolumn{7}{|l|}{ Carcinoma in situ } \\
\hline Invasive carcinoma & - & $1(1.9 \%)$ & - & $2(3.7 \%)$ & - & $3(5.6 \%)$ \\
\hline Total & $28(51.9 \%)$ & $19(35.3 \%)$ & $2(3.7 \%)$ & $4(7.4 \%)$ & $1(1.9 \%)$ & $54(100 \%)$ \\
\hline
\end{tabular}



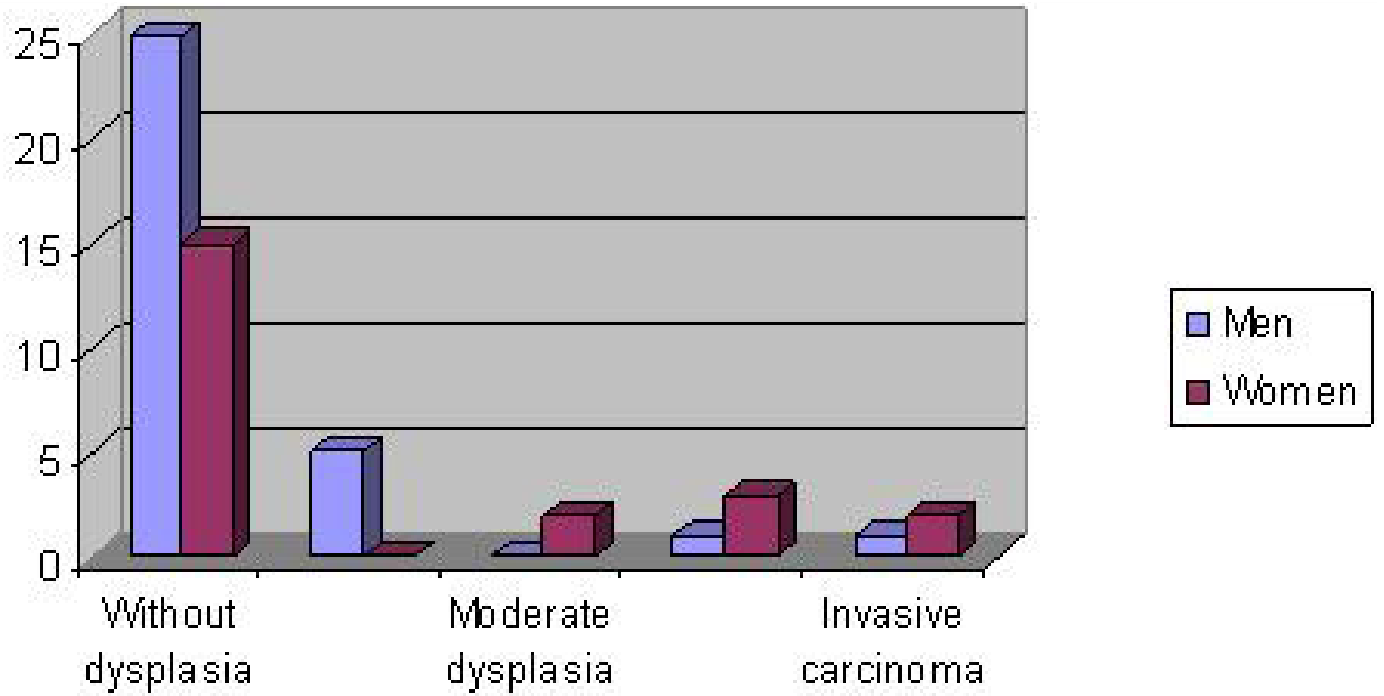

Fig. 3. Percentage association between degree of dysplasia and sex.

Table 2. Association between degree of dysplasia and lesion site

\begin{tabular}{|c|c|c|c|c|c|c|c|c|c|c|}
\hline \multirow{2}{*}{$\begin{array}{l}\text { Degree of } \\
\text { dysplasia }\end{array}$} & & \multicolumn{9}{|c|}{ Lesion site } \\
\hline & $\begin{array}{l}\text { Lateral } \\
\text { aspect of } \\
\text { tongue }\end{array}$ & $\begin{array}{l}\text { Ventral } \\
\text { aspect of } \\
\text { tongue }\end{array}$ & $\begin{array}{l}\text { Dorsal } \\
\text { aspect of } \\
\text { tongue }\end{array}$ & Gums & $\begin{array}{l}\text { Buccal } \\
\text { mucosa }\end{array}$ & $\begin{array}{c}\text { Behind } \\
\text { commissures }\end{array}$ & $\begin{array}{c}\text { Floor of } \\
\text { mouth }\end{array}$ & Palate & Lip & Total \\
\hline None & $6(11.1 \%)$ & $2(3.7 \%)$ & $2(3.7 \%)$ & $9(16.7 \%)$ & $7(13 \%)$ & $2(3.7 \%)$ & $4(7.4 \%)$ & $4(7.4 \%)$ & $4(7.4 \%)$ & $40(74.1 \%)$ \\
\hline Mild & $1(1.9 \%)$ & $2(3.7 \%)$ & - & - & $2(3.7 \%)$ & - & - & - & - & $5(9.3 \%)$ \\
\hline Moderate & $2(3.7 \%)$ & - & - & - & - & - & - & - & - & $2(3.7 \%)$ \\
\hline $\begin{array}{c}\text { Severe } \\
\text { dysplasia or } \\
\text { carcinoma } \\
\text { in situ }\end{array}$ & $3(5.6 \%)$ & - & - & - & - & - & $1(1.9 \%)$ & - & - & $4(7.4 \%)$ \\
\hline $\begin{array}{c}\text { Invasive } \\
\text { carcinoma }\end{array}$ & $2(3.7 \%)$ & - & - & $1(1.9 \%)$ & - & - & - & - & - & $3(5.6 \%)$ \\
\hline Total & $14(26 \%)$ & $4(7.4 \%)$ & $2(3.7 \%)$ & $10(18.5 \%)$ & $9(16.7 \%)$ & $2(3.7 \%)$ & $5(9.3 \%)$ & $4(7.4 \%)$ & $4(7.4 \%)$ & $54(100 \%)$ \\
\hline
\end{tabular}

\section{Discussion}

Our observation that leukoplakia was slightly more common in men than in women is consistent with the majority of reports from the literature (6-8), although there is a tendency for the difference in frequency between sexes to decrease (male to female ratio in our study, 1.45:1). This may be because smoking is becoming increasingly common in women. Like us, Scheifele et al (9) found that leukoplakias were diagnosed earlier in men than in women, although in our case the dif- ference was not statistically significant. In the study by Scheifele et al, conducted in the USA in 2003, the most common age group for the diagnosis of leukoplakia was 40-49 for men. In women, it was 70 years and above. In our series, the majority age group for diagnosis in both men and women was the sixth decade of life.

In our sample of 54 patients, $79.6 \%$ of the lesions were diagnosed in patients between the ages of 50 and 79. In a study conducted in India in 2005, however, Mishra et al (10) reported that the most common age group for the diagnosis of oral leukoplakia was 21-30, followed, in 
order, by the 31-40, 41-50, and 11-20 age groups. These data are striking as they contradict other reports. They might be due to lifestyle habits (fundamentally smoking, which is highly prevalent in India) and/or reflect idiosyncrasies of the sample.

Our findings are consistent with previous reports of both a cause-and-effect relationship between tobacco and leukoplakia (7,11-13) and a dose-response relationship (13). Indeed, it is well known that lesions improve in patients who quit smoking. Fisher et al (14), in contrast, in a case control study, did not find a significant association between oral leukoplakia and the daily use of alcohol and smoking, possibly because of an inadequate sample size. They did, however, find a significant association for chewing tobacco.

Morse et al (8) observed that alcohol consumption was more strongly associated with oral carcinoma than with epithelial dysplasia, particularly where drinks such as beer and spirits and high consumption levels were concerned. Our findings are consistent with these observations as in our series $55 \%$ of the patients did not drink and within the group of drinkers, only $6 \%$ drank more than $500 \mathrm{cc}$ of alcohol a day.

Dietrich et al (15) found a significant association between the prevalence of oral leukoplakia and diabetes mellitus. We were unable to confirm this association due to the small proportion of patients with diabetes in our series (5.6\%). Hypercholesterolemia and rheumatic diseases were the most common systemic diseases in our series. Consequently, perhaps the possible influence of hypercholesterolemia on the development of leukoplakia should be further investigated. Nonetheless, the high proportion of patients with hypercholesterolemia and rheumatic disease detected in our series might be simply due to the relatively advanced ages of the patients in our series as many of these diseases tend to occur in the later decades of life. It is also worthy of note that $35.2 \%$ of our sample had no systemic disease.

Scheifele et al (9) found that $52.3 \%$ of patients with homogeneous leukoplakia smoked; the corresponding percentage for those with non-homogeneous leukoplakia was $66.7 \%$. The percentage of smokers in the homogenous leukoplakia group in our series was significantly higher, at $46.43 \%$, contrasting with just $19.23 \%$ in the non-homogeneous leukoplakia group. Nevertheless, the association between smoking and lesion type was not statistically significant in our study.

Zhang et al (16) reported that certain areas of the oral cavity (the floor of the mouth, the ventral and lateral aspects of the tongue, and the soft palate) had greater malignant potential and that lesions located in low-risk zones were genetically different to those located in high-risk zones (containing genetic profiles associated with carcinoma in progression). We also believe that lesions in certain sites present a greater risk of malig- nant transformation as the carcinomas detected in our series were located on the lateral aspect of the tongue, the gums, and the floor of the mouth. Nonetheless, in a recent study of leukoplakia, Holmstrup et al (12) found that malignant potential was independent of lesion site. Mishra et al (10) observed that $0.54 \%$ of nodular leukoplakias, $15.2 \%$ of verrucous leukoplakias, and $20 \%$ of mottled leukoplakias were histologically diagnosed as malignant. In our series, the corresponding figures were $100 \%$ for erythroleukoplakias and $15.8 \%$ for verrucous leukoplakias, suggesting that certain clinical types of lesions have a tendency to become malignant. The association between type of lesion and degree of dysplasia in our series was statistically significant $(p=.007)$. It is widely accepted that epithelial dysplasia and the risk of malignant transformation are considerably less common in homogeneous leukoplakias, but this does not obviate the need for a biopsy.

Because the value of assigning a clinical score to degree of dysplasia has been questioned, a number of studies in recent years have been conducted to develop cellular and molecular markers capable of indicating the risk of malignant transformation of dysplastic epithelium and to predict behavior over time, something which cannot be done effectively with degree of dysplasia $(11,17)$. While promising, however, such techniques have still not succeeded in replacing conventional histopathology but they have gained a position as complementary methods. There is no doubt that advances in molecular biology will provide improved tools for the diagnostic and prognostic evaluation of dysplastic lesions. Even though the diagnosis of dysplasia is essentially subjective and certain recent studies have failed to find an association between degree of dysplasia and subsequent development of leukoplakia lesions, a histologic finding of epithelial dysplasia is currently considered to be the best indication of the existence of a greater risk of malignant transformation.

\section{Conclusions}

1. The most common lesion site in our series was the lateral aspect of the tongue.

2. Homogeneous leukoplakia with the most common clinical form.

3. Tobacco was the main causative agent identified but the role of alcohol should not be ignored.

4. Certain sites, such as the lateral aspect of the tongue, the floor of the mouth, and the gums, are associated with a greater risk of malignant transformation.

5. Erythroleukoplakias and verrucous leukoplakias have the greatest malignant potential. 


\section{References}

1. Axéll T, Pindborg JJ, Smith CJ, Van der Waal I. Oral white lesions with special reference to precancerous and tobacco- related lesions: conclusions of an international symposium held in Uppsala, Sweden, May 18-21 1994. International Collaborative Group on Oral White Lesions. J Oral Pathol Med. 1996;25:49-54.

2. Warnakulasuriya S, Johnson NW, Van der Waal I. Nomenclature and classification of potentially malignant disorders of the oral mucosa. J Oral Pathol Med. 2007;36:575-80.

3. Van der Waal I, Axéll T. Oral leukoplakia: a proposal for uniform reporting. Oral Oncol. 2002;38:521-6.

4. Silverman S Jr, Gorsky M, Lozada F. Oral leukoplakia and malignant transformation. A follow-up study of 257 patients. Cancer. 1984;53:563-8.

5. Van der Waal I, Schepman KP, Van der Meij EH. A modified classification and staging system for oral leukoplakia. Oral Oncol. 2000;36:264-6.

6. Alvarez Lorenzo M, Blanco Carrión A, Antúnez López J, Gándara Vila P, García García A, Gándara Rey JM. [Ultrastructural differences between leukoplakia with and without dysplasia]. Bull Group Int Rech Sci Stomatol Odontol. 2001;43:37-45.

7. Bánóczy J, Gintner Z, Dombi C. [Effect of smoking on the development of oral leukoplakia]. Fogorv Sz. 2001;94:91-6.

8. Morse DE, Psoter WJ, Cleveland D, Cohen D, Mohit-Tabatabai M, Kosis DL, et al. Smoking and drinking in relation to oral cancer and oral epithelial dysplasia. Cancer Causes Control. 2007;18:919-29.

9. Scheifele C, Reichart PA, Dietrich T. Low prevalence of oral leukoplakia in a representative sample of the US population. Oral Oncol. 2003;39:619-25.

10. Mishra M, Mohanty J, Sengupta S, Tripathy S. Epidemiological and clinicopathological study of oral leukoplakia. Indian J Dermatol Venereol Leprol. 2005;71:161-5.

11. Liu SC, Sauter ER, Clapper ML, Feldman RS, Levin L, Chen SY, et al. Markers of cell proliferation in normal epithelia and dysplastic leukoplakias of the oral cavity. Cancer Epidemiol Biomarkers Prev. 1998;7:597-603.

12. Holmstrup P, Vedtofte P, Reibel J, Stoltze K. Long-term treatment outcome of oral premalignant lesions. Oral Oncol. 2006;42:461-74.

13. Shiu MN, Chen TH, Chang SH, Hahn LJ. Risk factors for leukoplakia and malignant transformation to oral carcinoma: a leukoplakia cohort in Taiwan. Br J Cancer. 2000;82:1871-4.

14. Fisher MA, Bouquot JE, Shelton BJ. Assessment of risk factors for oral leukoplakia in West Virginia. Community Dent Oral Epidemiol. 2005;33:45-52.

15. Dietrich T, Reichart PA, Scheifele C. Clinical risk factors of oral leukoplakia in a representative sample of the US population. Oral Oncol. 2004;40:158-63.

16. Zhang L, Cheung KJ Jr, Lam WL, Cheng X, Poh C, Priddy R, et al. Increased genetic damage in oral leukoplakia from high risk sites: potential impact on staging and clinical management. Cancer. 2001;91:2148-55.

17. Santos-García A, Abad-Hernández MM, Fonseca-Sánchez E, Julián-González R, Galindo-Villardón P, Cruz-Hernández JJ, et al. Ecadherin, laminin and collagen IV expression in the evolution from dysplasia to oral squamous cell carcinoma. Med Oral Patol Oral Cir Bucal. 2006;11:E100-5.

\section{Acknowledgments}

This study was supported by FONACIT grant number 200500408 and CDCH UCV PG 10-006522-2006 